\title{
On the Problems and Countermeasures of the Warehousing and Distribution of Fresh products E-commerce
}

\author{
Yuqin Wang \\ Xi'an International University, Xi'an Shaanxi, 710077, China. \\ wyq-yr@163.com
}

\begin{abstract}
Fresh electric business is known as the "last blue ocean" of the development of the electricity supplier. The fresh electricity supplier's distribution is an important link in the development of fresh electric business. In recent years, the demand of urban residents to buy fresh products through the network platform has gradually strengthened, but the quality of fresh products caused by the factors of distribution, storage and other factors is more concerned by consumers, and also restricts the development of fresh electricity. In order to provide consumers with better customer experience, fresh electric business enterprises constantly innovate warehouse allocation mode to enhance the quality of warehouse service. On the basis of the analysis of the current status of the fresh electric business warehouse and distribution, this paper summarizes the existing problems in the storage and distribution of fresh electricity business, and further puts forward the countermeasures for the improvement of the warehouse and distribution of the fresh business.
\end{abstract}

Keywords: Fresh products E- commerce, Warehousing and distribution, "The last kilometer" distribution, the internet of things technology.

\section{Introduction}

In recent years, fresh electricity market in China has developed rapidly, but it is not a smooth sailing in the past ten years from the beginning to the present. All kinds of factors have made the fresh e-commerce difficulties, such as the consumers are still bound by the traditional fresh product purchase concept, the quality of fresh products, and the plight of logistics. This paper, combining the current situation of the development of fresh electricity, takes logistics warehousing and distribution as an entry point, analyzes the existing problems of storage and distribution, and seeks the improvement strategy of storage and distribution of fresh electricity, in order to solve the bottleneck of the development of fresh electric business, and provide suggestions for the development of fresh electricity business enterprises.

\section{The Status of Warehousing and Distribution of Fresh Electric Business}

China's fresh electricity market is developing rapidly, with an average annual growth rate of more than 50\%. In 2017, the market size was about 139 billion 130 million Yuan. According to the research data of the relevant agencies, it is shown that because of the unsound development of the cold-chain logistics and the difference of the people's consumption level, the coverage areas of Chinese fresh electricity are mainly concentrated in the first or second line cities, and the online shopping users are mainly in the first or second line cities. Among them, Beijing, Shanghai, Guangzhou and Shenzhen are the largest number of fresh online shopping users. The fresh electricity market is divided into three camps. The first camp is a comprehensive e-commerce platform represented by Tall and Dingdong. For example, Mall's meow fresh life channel and Dingdong's Dingdong home channel. The second camp is a vertical fresh electric business platform represented by Yugo, Tutu, etc. The third camp is based on Shun Feng as a representative of express cross boundary to fresh electricity supplier.

At present, the warehousing and distribution mode adopted by fresh electric business enterprises can be divided into three types: (1) self-built storage + self-distribution mode, this model is represented by Dingdong; (2) self-built storage + third party distribution mode, such as Belau, storage is self-construction, but distribution is cooperation with VTEPAI express Co. LTD. micro special ; 
(3) third party warehousing + distribution mode, Mall's "meow fresh life" is the typical use of this model.

\section{The Existing Problems in Warehousing and Distribution of Fresh Products E-Commerce}

\subsection{High Cost of Warehousing and Distribution of Fresh Products E-commerce}

In storage, on the basis of building a room temperature warehouse, the fresh electric business needs to be equipped with a professional standard of cold storage at different temperatures. The cost of storage is far higher than the storage cost of ordinary goods. In transportation vehicles, the transportation of fresh products needs refrigerated cars, the load of refrigerated cars is smaller than that of ordinary wagons, and the energy consumption is larger. These factors will increase the cost of transportation for Fresh products E-commerce. In the transfer center, in order to ensure the quality of the fresh products, the fresh products should be operated at the constant temperature operation, and the constant temperature operation is not only large in the early stage, but also the energy consumption is large and the cost increases. In the "last mile" distribution, fresh products need special packaging and incubators for double thermal insulation, which are much more expensive than ordinary express cartons.

\subsection{The Quality of the "Last Mile" Distribution Is Not High}

Fresh electricity supplier "last mile" distribution quality is not high and related to the following circumstances. First, the facilities and equipment for temporary storage of fresh products are not sound. In the actual distribution, it often occurs that customer is away when the courier sends the express to the customer. In the face of this situation, the courier often puts the parcel to the collection point near the District, the cell from the cabinet or the property duty room. These places are generally in the normal temperature state, which can easily cause the fresh products to be not fresh and affect the "last kilometer" distribution quality. Second, it is the poor timeliness of the last mile distribution. Some logistics companies will stipulate that fresh parcels should be given priority distribution, but the relevant systems are not perfect enough, and the couriers are not concerned about the timeliness of fresh products. During the delivery process, the courier will have his own plan to deliver the task as soon as possible, and distribute it in the district as a unit. Coupled with traffic congestion, weather and other uncontrollable factors, these may lead to fresh products cannot be delivered to consumers in accordance with the agreed time.

\subsection{Poor Traceability of Warehouse Distribution Information}

The application of modern information management means is not enough in the warehousing and distribution of fresh electronic business. At present, the information exchange of logistics company nodes depends mainly on the information uploaded by the terminal scanning equipment. It can be divided into three scanning processes. One package is scanned at the first time when the package is sent, the second scan in the distribution center and it is scanned for the last time after the customer has signed the package. During the whole transportation process, there is a "blind spot" in the monitoring of fresh products, such as the final delivery, which leads to the failure to achieve the whole process monitoring and effective tracing of fresh products.

\section{Improvement Measures of Warehouse and Distribution of Fresh Products E- Commerce}

\subsection{Training Localization Ability and Strengthening Cost Control of Warehouse and Distribution}

At present, the core problem that the fresh electricity supplier needs to solve is the logistics problem, hoping to make the low cost fresh logistics possible. As the saying goes, eat in the local and 
eat in the season. The fresh products of local production and local supply are not introduced in the field. The fresh electricity suppliers can cooperate with the local fresh product suppliers, and also cooperate with the local logistics enterprises to complete the local distribution. In the current environment, the fresh electricity business can also cooperate with the local fresh supermarket, convenience store and so on. It will be able to meet the requirements of the elderly and provide convenience for the busy working people.

\subsection{Set up Common Community Service Points and Optimize "Last Mile"}

"The last mile" distribution is a big problem for Fresh products E-commerce. In recent years, many fresh electric business enterprises rely on offline outlets to solve the problem. However, the offline stores belong to a fresh business platform, and there is no information communication between the offline stores of different platforms, which affects the formation of the distribution scale and the high cost. According to the particularity of the distribution of fresh products, community service can be established in community as a community service point, and a community service point can include a number of logistics companies or a number of fresh electric business platforms. Community service points integrate the demand of the dispersed residents to the delivery time, and submit the information to the logistics company or the fresh business platform, and then distribute the information according to the integrated information. This method provides convenience for distribution and improves distribution efficiency.

\subsection{Making Full Use of Internet of Things Technology to Achieve Full Process Control}

With the rapid development of the society, consumers have put forward higher requirements for the quality of fresh products. The traditional logistics mode has not been fully guaranteed. It is necessary to make full use of new technologies to ensure the quality of fresh products and make the whole logistics process more transparent.

\subsubsection{Tracking the Fresh Products Through the Whole Course}

With the technology of Internet of things, the information of the production, processing, storage and distribution of fresh products is integrated to realize the whole course of the fresh products "from the field to the table". In the production stage of fresh products, record management is carried out. The records of product origin information, production process information and packaging information are recorded and uploaded to the database. After the fresh products are processed and packaged, the staff will update the product processing and distribution information and upload the system, and then generate two-dimensional code labels and print and paste on the packaging of fresh products. In the distribution process of fresh products, all the information of the product can be obtained by scanning the two-dimensional code labels on the packaging. The concept of "construction package" is used to bind the fresh product and vehicle information, to locate the vehicle in real time through $3 \mathrm{~S}$ technology. At the same time, the administrator of the supervision center can send the query, control and scheduling instructions to the driver through the vehicle system, so as to realize the real-time monitoring of the fresh products in the transportation and distribution links. Consumers can understand the information of each link by scanning the two-dimensional code on the fresh product packaging, and then sign the products.

\subsubsection{Full Control of Temperature}

The whole process control of fresh products from production processing to "last mile" distribution temperature will help maintain the freshness of fresh products. Especially in the transportation process, the record of fresh products' status is helpful to inquire about the reason of freshness preservation and enhance the service of fresh electronic business.

In the course of transportation, the temperature and humidity of vehicles and the driving route of vehicles are monitored in real time by RFID technology. The temperature and humidity label combines RFID and sensing technology. Besides identifying and locating functions, it can also acquire temperature and humidity data and record in real time. The temperature and humidity recorder transfers the temperature change inside the carriage to the temperature control center. Through this 
data, the temperature changes during transportation can be judged, and timely measures should be taken according to the change of temperature and humidity, so as to maintain the quality of fresh products. In the background, the data recorded by RFID can be transmitted to the fresh electronic business platform and consumers through data transfer and transformation system.

\subsection{Actively Carry Out the Innovation of the Warehouse Distribution Pattern}

Because of the particularity of the fresh products, we can adopt the model of "the direct production of the origin and the fresh cloud storehouse of the community". This model has the nature of localization and group buying, which is more suitable for people's living habits. The direct supply mode can shorten the journey time of fresh products and ensure the quality of products. Moreover, the reduction of intermediate logistics links can reduce the cost of fresh electronic business distribution and make certain advantages in selling prices. After the consumer place an order in online platform, the platform's customer service personnel will integrate the customer's orders, purchase directly in the origin and distribute it to the consumers, so that the intermediate links can be saved and the quality of the products is guaranteed. The fresh business platform can also establish a cooperative relationship with the local farm or planting base, allowing customers to receive fresh products from the field in the shortest possible time.

Fresh electric business enterprises can use the concept of rookie operation, and cooperate with the community as a unit to establish community fresh cloud storage. The community fresh cloud storehouse mainly has two functions, one of which is to integrate resources. The community fresh cloud storehouse uses the Internet to integrate the scattered customer resources, including the customer's receiving time, commodity types and so on. At the same time, the community fresh cloud storehouse also has the functions of door-to-door delivery, self-introduction, and return and so on. After the success of the order, customers can choose to go to the community to pick up their items after work, or make an appointment for a suitable time to send the fresh products to the door free of charge by the distributor. If consumers are not satisfied with the product, they can directly return the product to the community fresh cloud storehouse, and no longer need to find the logistics company to refund it. For the returned products, the community fresh cloud storehouse will be refunded or sold locally according to the actual situation.

\section{Conclusion}

In recent years, the fresh electricity supplier has blowout development status, known as the "last blue ocean" of the electricity supplier industry. However, the quality of fresh products, distribution, warehousing and other factors, the development of fresh electricity providers still have many difficulties. The fresh electric business needs to directly face the problem of the warehouse, take full consideration of the factors such as the fresh product itself, the particularity of the electric business, the cold chain logistics and other factors, actively carry out the innovation of the warehouse distribution mode, and promote the construction of information and the whole process of cold chain logistics, in order to improve the storage efficiency of fresh products, reduce the cost of warehousing, ensure the quality and safety of fresh products.

\section{Acknowledgments}

This paper is the social science fund project of Shaanxi province in 2016, Serial No.2016R045.

\section{References}

[1]. Wang Lin, Zhao Yu, Fu Xiaojie. Study on Last Mile Distribution of Fresh Produce E-businesses. Logistics Technology, 2016, 35(06), 12 15.

[2]. Fan Xueqian. Analysis of Distribution Modes of Fresh Product E-businesses: A Case Study on Sfbest.com. Logistics Technology, 2017, 36(08), 40 43. 
[3]. Xu Guangzhou, Song Zigong. Coordinating Contract between Fresh Agricultural Products Ebusiness Enterprise and Logistics Service Provider--An Analysis based on Fresh Agricultural Products Home Delivery Mode. Commercial Research, 2017, 02), 151 157.

[4]. Zhang Xiamen. Current Situation, Problems and Development Trend about the Logistics of Fresh Product E-commerce. Huizhou Agricultural Sciences, 014, 2(11), 275 278.

[5]. Zhang Xiamen. Source and Formation Mechanism of Logistics Risk about Fresh Product Ecommerce Under Perspective of Supply Chain. Agricultural Economics and Management, 2015, 03), $1 \sim 87$. 